\title{
Influence of Chain Stiffness on the Interaction of Polyelectrolytes with Oppositely Charged Micelles and Proteins ${ }^{\dagger}$
}

\author{
A. B. Kayitmazer, E. Seyrek, P. L. Dubin,* and B. A. Staggemeier \\ Department of Chemistry, Indiana-Purdue University, Indianapolis, Indiana 46202
}

Received: January 10, 2003; In Final Form: May 2, 2003

\begin{abstract}
The effect of a polyelectrolyte's chain stiffness on its interaction with an oppositely charged colloid particle was studied by measuring the relative affinity of two polyelectrolytes for (1) mixed cationic/nonionic micelles (DTAB/TX100), and (2) the protein serum albumin. The binding affinity as manifested, respectively, in the critical ionic surfactant mole fraction required for polyelectrolyte-micelle complex formation, and in the critical $\mathrm{pH}$ for polyelectrolyte-protein association, was determined by turbidimetric titrations over a range of ionic strengths. Binding was generally weaker for the stiffer chain, hyaluronic acid (HA), relative to the more flexible chain, a copolymer of acrylamidomethylpropanesulfonate (AMPS) and acrylamide (AAm), chosen to have the same linear charge density as $\mathrm{HA}$ at neutral $\mathrm{pH}$. In the case of serum albumin, comparisons were also made to AMPS - AAm copolymers of higher charge densities, and to heparin, a highly charged and flexible biopolyelectrolyte. The results are discussed in terms of the ionic strength dependence of the relevant persistence lengths.
\end{abstract}

\section{Introduction}

Ionic and mixed ionic/nonionic surfactants aggregate to form charged micelles, which may associate with oppositely charged polyelectrolytes (PE) to form micelle-polyelectrolyte complexes. ${ }^{1-7}$ The evidence is strong that polyelectrolyte binding to monomeric surfactants is negligible when the surfactant concentration is large compared to the critical micelle concentration $(\mathrm{cmc})$, so the association is predominantly an electrostatic interaction between polymer and micelle, in contradistinction to the binding of surfactant monomers and clusters at concentrations well below the cmc, e.g., at the "critical aggregation concentration (cac)". ${ }^{8}$ The interaction between polyelectrolytes and oppositely charged micelles has received substantial attention, initially perhaps related to some specific industrial applications, but subsequently as a model for interactions between polyelectrolytes and oppositely charged colloids in general, with implications for a wide range of systems, including such diverse phenomena as colloid flocculation in water clarification ${ }^{9}$ and nonspecific binding of histones and other proteins to DNA. ${ }^{10,11}$ These systems are complicated because the binding behavior is affected by so many variables related to polyelectrolyte structure, surfactant composition, and the solvent medium, and thus including (i) polyelectrolyte composition, charge density, chain length, and chain flexibility; (ii) micelle surface charge density, micelle size, and shape; and (iii) $\mathrm{pH}$ and ionic strength of the medium.

The preceding list of variables would seem to pose a daunting problem for the selection of experimental conditions, and this does explain why comparisons among experimental studies are difficult. Fortunately, three of these variables have a dominant effect on the energetics of $\mathrm{PE}$-micelle complexation: ionic strength $I$, polymer linear charge density $\xi$, and micelle surface charge density $\sigma .^{1-7}$ The central role of these parameters is

\footnotetext{
† Part of the special issue "International Symposium on Polyelectrolytes".

* To whom correspondence should be addressed. E-mail: dubin@chem. iupui.edu.
}

consistent with theories of polyelectrolyte-colloid interactions. ${ }^{12-15}$ Mixed ionic-nonionic micelles provide a convenient way to control $\sigma$ via the stoichiometric mole fraction of the ionic component $(Y)$. The observation of critical values of $Y$ at the point of incipient complex formation, and their simple correlation with $I$ and $\xi{ }^{16}$ suggests that the ability of the polyelectrolyte to alter the composition and size of the mixed micelles, while possibly not negligible, appears to be a secondary effect, at least at conditions close to incipient binding. While $Y_{\mathrm{c}}$ depends strongly on ionic strength, it is essentially independent of total surfactant concentration, polymer concentration, and polymer chain length, ${ }^{5}$ suggesting that $Y_{\mathrm{c}}$ reflects the process in which one micelle binds to some portion of the polyelectrolyte chain. ${ }^{2}$ As noted above, the dependence of $Y_{\mathrm{c}}$ on $I$ and $\xi$ can productively be viewed in the context of several theories that predict such an "adsorption-desorption transition" for polyelectrolytes in contact with oppositely charged planar or spherical surfaces. ${ }^{14}$

The binding of polyelectrolytes to proteins ${ }^{17,18}$ is also predominantly governed by electrostatic forces. Park et al. ${ }^{17 a}$ observed that "primary" soluble protein-polyelectrolyte complexes, defined as a single polyelectrolyte with multiple bound proteins, form at a critical $\mathrm{pH}\left(\mathrm{pH}_{\mathrm{c}}\right)$, which was found to be a function of the ionic strength and the linear charge density of the polyelectrolyte. ${ }^{18 \mathrm{~b}, \mathrm{c}}$ As the $\mathrm{pH}$ is gradually changed (increased in the case of polycations and decreased for polyanions), the protein gains charge opposite that of the polyions, so that the complex charge may approach electroneutrality, allowing for higher order association. While $\mathrm{pH}_{\mathrm{c}}$ is analogous to $Y_{\mathrm{c}}$, it is notable that binding often occurs "on the wrong side of the isoelectric point", e.g., at $\mathrm{pH}>\mathrm{p} I$ for proteins binding to polyanions. ${ }^{17}$ This is because "charge patches" on the protein surface, opposite in sign to the net protein charge $\left(Z_{\mathrm{p}}\right)$, can provide sites for the binding of polyelectrolytes having the same sign as $Z_{\mathrm{p}}$. The size of the binding patch on the protein surface is determined by PE chain flexibility and linear charge density. ${ }^{18 \mathrm{~b}, \mathrm{c}}$ 
Several workers have examined complexation between polyelectrolytes and colloidal macroions using Monte Carlo simulations. ${ }^{19-22}$ Wallin and Linse ${ }^{19}$ considered the effect of polyion chain flexibility for the interaction of a charged micelle with one oppositely charged polyelectrolyte. Simulations showed a very large accumulation of the polyelectrolyte charges close to the micelle for a flexible polyelectrolyte, which decreased strongly at some increased polymer chain rigidity. A large decrease in the chain conformational entropy thus resulted in a less tight complex with the micelle. Skepo and Linse ${ }^{21}$ examined the effect of salt concentration on the complexation of several macroions with one polyelectrolyte at varying flexibility. According to their simulation results, as the salt concentration is increased the number of bound PE segments near a charged macroion is found to decrease substantially for the flexible chain while it remains constant but smaller for the stiff one. Stoll and Chodanowski ${ }^{22}$ also used Monte Carlo simulations to study the effect of chain flexibility on the complexation of a polyelectrolyte with an oppositely charged colloid. They concluded that an increase in the chain flexibility and/or a decrease in the salt concentration enhance polyelectrolyte adsorption. They defined a critical ionic concentration at which adsorption/desorption is observed and found it to depend on the polyelectrolyte bare persistence length $\left(l_{\mathrm{p}}{ }^{\circ}\right)$.

While simulations and theory have explicitly considered the effect of chain stiffness, there are few experimental reports on the effect of polymer flexibility on polyelectrolyte-colloid (e.g., micelle) complexation. References 19 and 20 refer to experiments related to the effect of the persistence length on the "cac", but since the cac is a discontinuity observed far below the $\mathrm{cmc}$, such experiments are at best an indirect test of the simulations. Our goal in the present study is to investigate experimentally the effect of PE chain stiffness on complexation. Since chain rigidity often goes hand-in-hand with insolubility, or else arises from helix formation, it is difficult to vary chain stiffness without changing other structural features. Here we use two polyanions: a flexible random copolymer of AMPS (acrylamidomethylpropanesulfonate) and acrylamide ( $80 \mathrm{~mol} \%$ acrylamide), and the relatively stiff carboxylated biological polysaccharide, hyaluronic acid (HA), ${ }^{23}$ both of which have the same rather low charge density. The structural charge density of HA is $\xi=$ 0.6 , where $\xi$, the Manning counterion condensation ${ }^{24 a}$ parameter, is unity when the average distance between charge groups is equal to the Bjerrum length, $7 \AA$ in water. While this would correspond to $25 \mathrm{~mol} \%$ AMPS, results reported elsewhere suggest that high-conversion random ionic-nonionic copolymers display, in their binding to oppositely charged colloids, effective charge densities slightly larger than their average compositions would indicate, because the onset of complexation is governed by residue sequences enriched in the ionic monomer residue ${ }^{16}$ for this reason we employ a $20 \%$ AMPS copolymer. The ionic strength dependence of the persistence length of the copolymer $\left(\mathrm{AMPS}_{17} / \mathrm{AAm}_{83}\right.$ ) has been reported by Reed et al., ${ }^{25}$ and its bare persistence length $l_{\mathrm{p}}{ }^{\circ}$ falls within the range of values reported for the two corresponding homopolymers, i.e., between 2 and $3 \mathrm{~nm}$. For hyaluronic acid (HA), values for $l_{\mathrm{p}}{ }^{\circ}$ ranging from 4 to $9 \mathrm{~nm}$ have been reported $;{ }^{26-30}$ here we use the values closer to $4 \mathrm{~nm}$ of Hayashi et al., ${ }^{27,28}$ who have argued that the larger $l_{\mathrm{p}}{ }^{\circ}$ values reported elsewhere arise from a significant effect of the excluded volume on the $z$-average radius of gyration. Thus, the difference in chain stiffness here may be small as $35 \%$, but as we shall see, this leads to significant effects.

The effects of chain stiffness on polyelectrolyte-colloid binding can be observed in various ways. Muthukumar obtained explicit analytical solutions at the onset of binding that relate the variables corresponding to ionic strength $I$, temperature, polymer charge density, polymer molecular weight, and colloid radius and surface charge density at the adsorption-desorption transition. ${ }^{14}$ As noted, the simulations of Stoll and Chodanowski show a desorption-adsorption transition when $I$ exceeds some value that depends on the other variables mentioned above. ${ }^{22}$ However, other simulations disclose no transitions with changing $I$ but instead a change in the number of polymer residues residing in the vicinity of the colloid surface. ${ }^{19-22,31}$ What we observe experimentally is a transition from noninteracting to complexed states at fixed ionic strength, upon increase in the surface charge density of a cationic-nonionic mixed micelle in the presence of HA or $\mathrm{AMPS}_{20} / \mathrm{AAm}_{80}$.

Mixed micelles provide a system in which a homogeneous spherical colloid surface charge density can be varied continuously, but the lability of surfactant monomers may raise questions about micelle reorganization. No such factors are at play with proteins, whose surface charge densities can be controlled by $\mathrm{pH}$ (although, as noted above, the charge heterogeneity and asymmetry of proteins makes comparison with theory quite difficult). Furthermore, the interactions of proteins with biopolyelectrolytes are of considerable biochemical relevance, a case in point being the putative binding of serum albumin to HA in synovial fluid, and its possible role in joint malfunction. ${ }^{32}$ For this reason, we also report on comparison of HA and $\mathrm{AMPS}_{x} / \mathrm{AAm}_{y}$ binding to serum albumin (SA). This study is supplemented by experiments in which the critical $\mathrm{pH}$ for complexation is determined also for heparin, a high-chargedensity flexible chain biopolyelectrolyte.

\section{Experimental Section}

Materials. Sodium hyaluronate $(\mathrm{Na}-\mathrm{HA}, \mathrm{MW}=900 \mathrm{~K}$ and $1200 \mathrm{~K}$ ) was obtained from Shiseido Research Center (Yokohama). A random copolymer of 2-acrylamido-2-methylpropanesulfonate (AMPS) and acrylamide (AAm) was prepared ${ }^{33}$ by free-radical copolymerization in dimethylformaldehyde (DMF) with 2:8 mole ratio AMPS:AAm. After purification by precipitation $(2 \times)$ in acetone, dialysis $(24 \mathrm{~h})$, and freeze-drying, dynamic light scattering (Dynapro $801,0.1 \mathrm{M} \mathrm{NaCl}$ ) gave an apparent Stokes radius of ca. $20 \mathrm{~nm}$, corresponding to an approximate molecular weight on the order of several hundred thousand. Since the binding energy of ionic/nonionic micelles to polyelectrolytes has been observed, for $\mathrm{MW}>10^{4}$ to be independent of polymer molecular weight, ${ }^{34,35}$ no further molecular weight characterization was done. $\mathrm{AMPS}_{25} / \mathrm{AAm}_{75}$ and $\mathrm{AMPS}_{80} / \mathrm{AAm}_{20}$ random copolymers (MW 200 000), used to determine $\mathrm{pH}_{\mathrm{c}}$ for bovine serum albumin (BSA) and human serum albumin (HSA), were prepared by Takeshi Sato (Osaka University). Standard heparin (98H0713) with molecular weight 18000 was purchased from Sigma Chemical Company and used without further purification.

Triton X-100 (TX100) and dodecyltrimethylammonium bromide (DTAB, purity 99\%) were purchased from Sigma-Aldrich. $\mathrm{NaCl}$, sodium phosphate monobasic, and sodium phosphate dibasic anhydrous were purchased from Fisher Scientific. BSA (24H0177) and HSA (66H9306) were obtained from Sigma Chemical Co (St. Louis, MO). All were used without further purification. Milli-Q water (filtered with $0.22 \mu \mathrm{m}$ Millipak 40, Millipore) was used in all experiments.

All polymers and surfactants were dissolved in a 1:9 (mole/ mole) mixture of phosphate buffer- $\mathrm{NaCl}$ solution at the desired total ionic strength, adjusted to a $\mathrm{pH}$ between 6.5 and 7.5. At this $\mathrm{pH}$, the carboxylic groups of HA are fully ionized. HA 

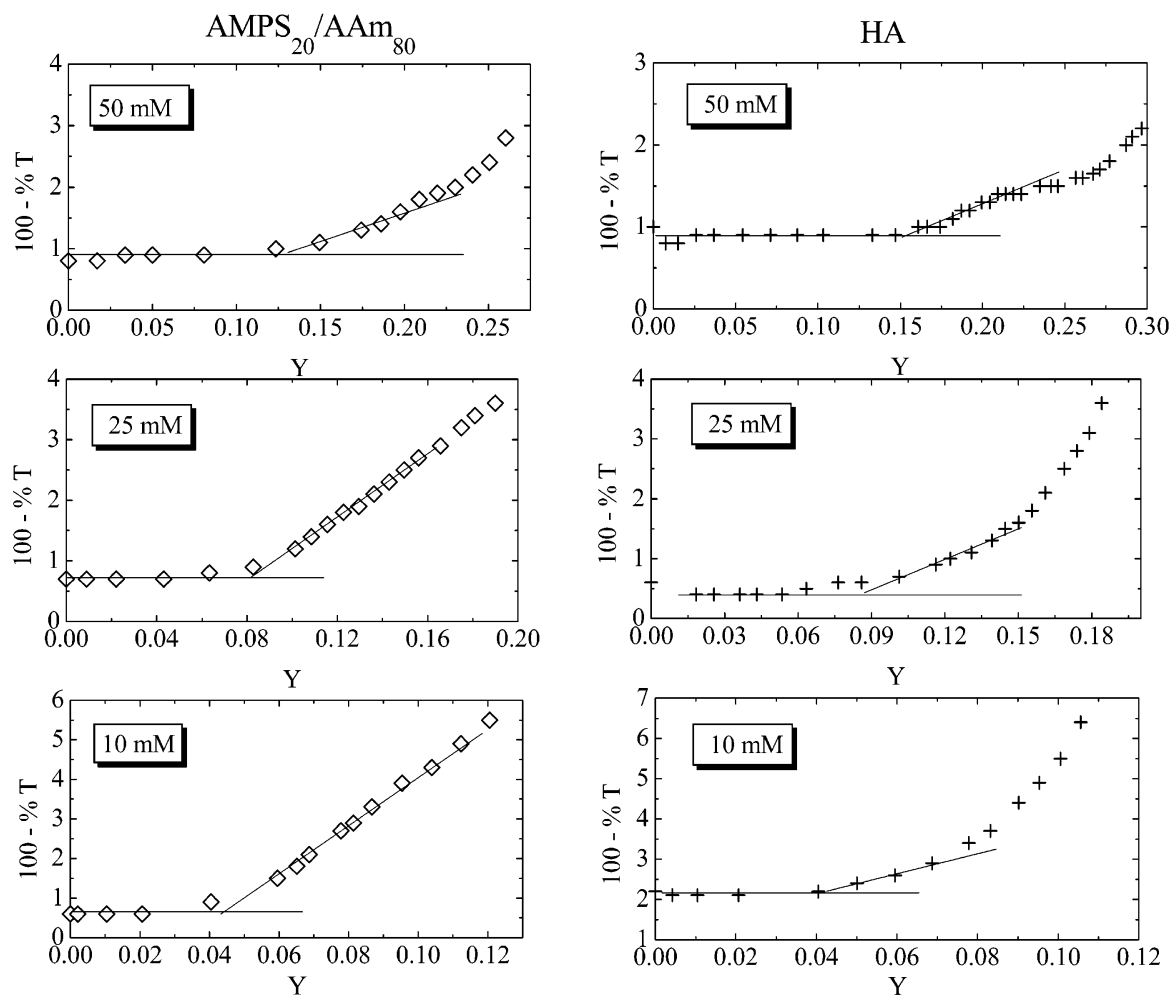

Figure 1. Turbidity $(100-\% T)$ as a function of anionic surfactant composition $(Y)$ in the presence of either HA $(+)$ or AMPS $20 / \mathrm{AAm}_{80}(\diamond)$, in 10, 25, and $50 \mathrm{mM}$ 9:1 NaCl:phosphate $\mathrm{pH} 6.5$ buffer.

solutions were stirred overnight to ensure complete solubilization. All other polymer solutions were dissolved for at least 2 h. The solutions were filtered using $0.22 \mu \mathrm{m}$ cellulose acetate membranes (Sartorius, Viva Science Inc.).

Turbidimetric Titrations. Turbidimetric measurements, reported as $100-\% T$, were performed with a Brinkmann PC800 colorimeter $(\lambda=450 \mathrm{~nm})$ with a $2.0 \mathrm{~cm}$ path length fiber optics probe. In "Type 1 " titrations for investigation of micelle/polyelectrolyte critical binding conditions, DTAB was added at constant ionic strength, with continuous stirring, to solutions of polymer (HA or AMPS $20 / \mathrm{AAm}_{80}$ ) and TX100 with initial concentration of $0.5 \mathrm{~g} / \mathrm{L}$ polymer and $20 \mathrm{mM}$ TX100. The mole fraction of DTAB

$$
Y=\frac{[\mathrm{DTAB}]}{[\mathrm{DTAB}]+[\mathrm{TX} 100]}
$$

controls the micelle surface charge density and thus its electrostatic interaction with the polyanion. It has been amply demonstrated that the critical values of $Y_{\mathrm{c}}$ or $\mathrm{pH}_{\mathrm{c}}$ determined by turbidimetry are in good agreement with values obtained from other parameters, such as the diffusion coefficient from dynamic light scattering or the mobility from electrophoretic light scattering.

Dependence of the turbidity on the $\mathrm{pH}$ ("Type 1" proteinpolyelectrolyte titration) was obtained by $\mathrm{HCl}$ addition to solutions of $0.5 \mathrm{~g} / \mathrm{L}$ serum albumin and $0.05 \mathrm{~g} / \mathrm{L}$ polymer (HA, heparin, and $\mathrm{AMPS}_{25} / \mathrm{AAm}_{75}$ or $\mathrm{AMPS}_{80} / \mathrm{AAm}_{20}$ ) in varied concentrations of $\mathrm{NaCl}$. The $\mathrm{pH}$ was monitored with a Corning 240 meter equipped with an Orion combination probe. Polymerfree blanks were used to eliminate the effect of free protein scattering.

\section{Results and Discussion}

Polyelectrolyte-Micelle Interaction. Turbidimetric titration curves for systems of TX100/DTAB-HA or TX100/DTAB-
$\mathrm{AMPS}_{20} / \mathrm{AAm}_{80}$ at ionic strengths (I) ranging from 10 to 400 $\mathrm{mM} \mathrm{NaCl} /$ phosphate buffer solution are presented in Figures $1-3$ as the dependence on $Y$ of $\tau=100-\% T$, with a precision of $\pm 0.2 \% T$. The onset of reversible soluble complex formation, $Y_{\mathrm{c}}$, was identified as the point at which $\mathrm{d} \tau / \mathrm{d} Y$ becomes greater than zero. At $I<100 \mathrm{mM}$, an abrupt increase in turbidity with values of $\% T<70$ was observed, indicating the onset of macroscopic phase separation. While the value of $\tau$ for a polymer-free solution ("blank") was found to show a shallow minimum as a function of $Y$, its slope was essentially negligible over small ranges of $Y$, so that no "blank correction" was made. The slope for the polymer-micelle solutions was always zero at low $Y$, but the small values of $\mathrm{d} \tau / \mathrm{d} Y$ above $Y_{\mathrm{c}}$ at some conditions, particularly at high $I$, required particular care in the determination of $Y_{\mathrm{c}}$. Plots at three consecutive ionic strengths were overlaid to ensure consistency in fitting the data points to straight lines. It should be noted that the digital signal of the colorimeter leads to meaningless apparent plateaus in, e.g., the highly expanded plots for HA at highest $I$, which would not be observed with an analog turbidity signal. Duplicate measurements did show that $Y_{\mathrm{c}}$ could typically be determined with a reproducibility of better than $2 \%$ relative except for the measurements for HA at high $I$.

With increasing ionic strength, the turbidities above $Y_{\mathrm{c}}$ diminish, along with a corresponding diminution in $\mathrm{d} \tau / \mathrm{d} Y$, most dramatically for HA, for which turbidity increases of less than $0.5 \% T$ are seen. As noted above, phase separation can be observed for both polymers at $I<100 \mathrm{mM}$; however, no phase separation was observed at any value of $I>100 \mathrm{mM}$ for HA and at any value of $I>175 \mathrm{mM}$ for $\mathrm{AMPS}_{20} / \mathrm{AAm}_{80}$. These results indicate that the nature of the soluble complex changes with increasing ionic strength. If phase separation is a consequence of charge neutralization via complexation, one may speculate that the requisite charge complementarity is impossible when high charge density micelles interact with low charge 

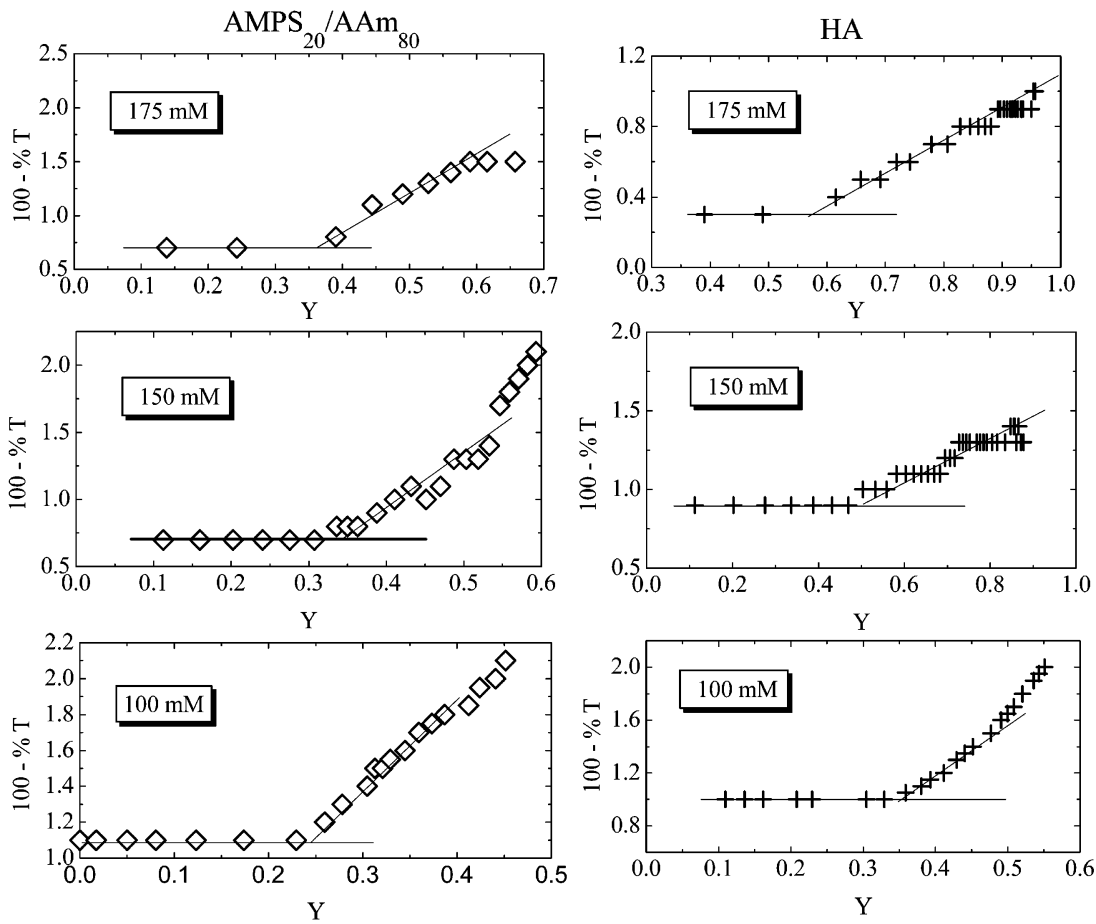

Figure 2. Turbidity as a function of $Y$ in the presence of either HA $(+)$ or $\mathrm{AMPS}_{20} / \mathrm{AAm}_{80}(\diamond)$, in 100, 150, and 175 mM 9:1 NaCl:phosphate pH 6.5 buffer.
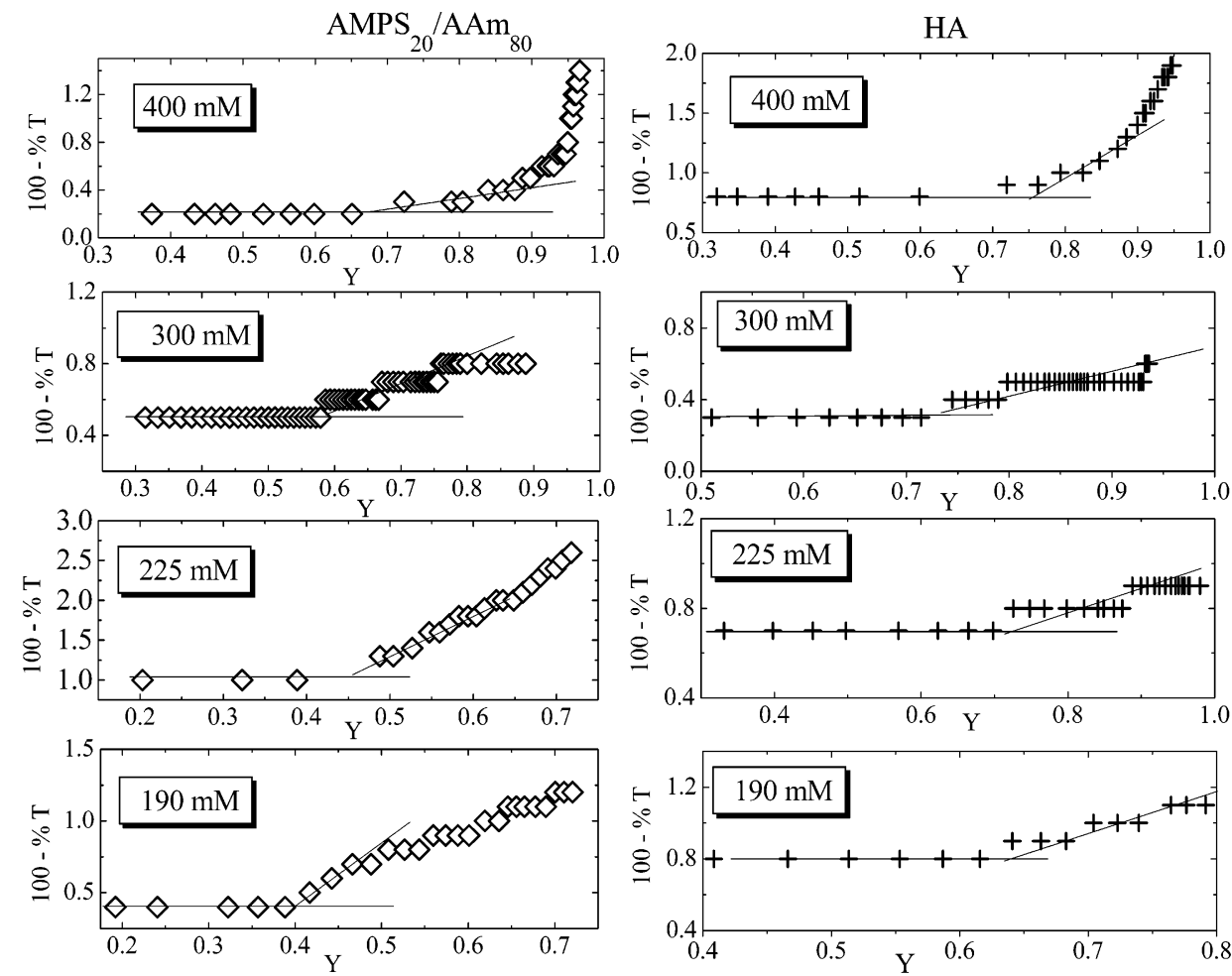

Figure 3. Turbidity as a function of $Y$ in the presence of either HA (+) or $\mathrm{AMPS}_{20} / \mathrm{AAm}_{80}(\diamond)$, in 190, 225, 300, and 400 mM 9:1 NaCl:phosphate pH 6.5 buffer.

density polymers, as is the case at high $I$. The subtle changes in turbidity in the vicinity of $Y_{\mathrm{c}}$ may also serve to point out that the onset of complexation is by no means a first-order transition. Regardless of these points, $Y_{\mathrm{c}}$ can be used to assess the relative micelle-binding affinities of HA and $\mathrm{AMPS}_{20} /$ $\mathrm{AAm}_{80}$, the main focus of the current work. The values of $Y_{\mathrm{c}}$ for $\mathrm{HA}$ and $\mathrm{AMPS}_{20} / \mathrm{AAm}_{80}$ are given in Table 1 for ionic strengths ranging from 10 to $300 \mathrm{mM}$. Plots of $Y_{\mathrm{c}} \mathrm{vs} I$, shown in Figure 4, may be viewed as "phase boundaries" as they separate conditions for complex formation from noninteracting domains. Binding of cationic micelles to $\mathrm{AMPS}_{20} / \mathrm{AAm}_{80}$ occurs more readily (i.e., at lower $Y_{\mathrm{c}}$ ) relative to that for $\mathrm{HA}$ at $I>$ ca. $75 \mathrm{mM}$, and the behavior of the two polymers converges at lower ionic strengths.

The phase boundary in Figure 4 defines the conditions for complex formation in terms of the ionic strength and the ionic surfactant composition, $Y$. Since $Y$ is the fraction of micelle headgroups that bear a charge, it is - to a first approximation 
TABLE 1: Ionic Strength Dependence of the Critical Conditions for Binding of TX100/DTAB Micelles to HA and $\mathbf{A M P S}_{20} / \mathbf{A A m}_{\mathbf{8 0}}$

\begin{tabular}{lll}
\hline & \multicolumn{2}{c}{$Y_{\mathrm{c}}$} \\
\cline { 2 - 3 }$I(\mathrm{M})$ & $\mathrm{AMPS}_{20} / \mathrm{AAm}_{80}$ & \multicolumn{1}{c}{$\mathrm{HA}$} \\
\hline 0.010 & $0.042 \pm 0.01$ & $0.045 \pm 0.002$ \\
0.025 & $0.085 \pm 0.005$ & $0.088 \pm 0.003$ \\
0.050 & $0.12 \pm 0.03$ & $0.155 \pm 0.005$ \\
0.100 & $0.24 \pm 0.01$ & $0.35 \pm 0.01$ \\
0.150 & $0.34 \pm 0.02$ & $0.50 \pm 0.01$ \\
0.175 & $0.36 \pm 0.01$ & $0.56 \pm 0.01$ \\
0.190 & $0.40 \pm 0.01$ & $0.625 \pm 0.005$ \\
0.225 & $0.46 \pm 0.01$ & $0.71 \pm 0.01$ \\
0.300 & $0.584 \pm 0.004$ & $0.73 \pm 0.01$ \\
0.400 & $0.67 \pm 0.02$ & $0.75 \pm 0.02$
\end{tabular}

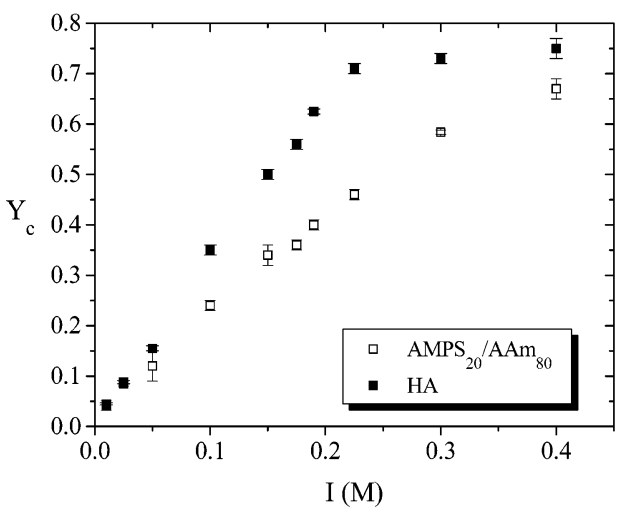

Figure 4. Critical micelle compositions at the point of onset of binding $\left(Y_{\mathrm{c}}\right)$ for TX100/DTAB mixed micelles, in the presence of either hyaluronic acid (ם) or $\mathrm{AMPS}_{20} / \mathrm{AAm}_{80}(\square)$, in 9:1 NaCl:phosphate $\mathrm{pH}$ 6.5 buffer, as a function of ionic strength.

- linear with the micelle surface charge density $\sigma$. From dynamic light scattering we find that the micelle diameter $d_{\mathrm{m}}$ at $I=0.20 \mathrm{M}$ changes from 4.0 to $3.0 \mathrm{~nm}$ as $Y$ goes from 0.5 to 1 , with an identical change over the range of $Y_{\mathrm{c}}$ values for HA from 0.10 to $0.4 \mathrm{M}$. The values of $d_{\mathrm{m}}$ in $I=0.1 \mathrm{M}$ at the respective $Y_{\mathrm{c}}$ values of HA and copolymer are 4.0 and $4.6 \mathrm{~nm}$. To avoid comparison of HA and the copolymer under situations where, at some given ionic strength, the micelle size at $Y_{\mathrm{c}}$ for $\mathrm{HA}$ is different from the micelle size at $Y_{\mathrm{c}}$ for the copolymer, further analysis of data is restricted to the range $0.04<Y<$ 0.7 in which the micelle size remains between 30 and $45 \mathrm{~nm}$. In this range, where micelle aggregation number is not strongly dependent on $Y$, we can have more confidence in the assumptions (1) that $Y_{\mathrm{c}}$ and $\sigma$ are linearly related, and (2) that the difference in micelle binding between $\mathrm{HA}$ and $\mathrm{AMPS}_{20} / \mathrm{AAm}_{80}$ as measured by $Y_{\mathrm{c}}$ is not complicated by a difference in micelle size.

The simple linear relationship between $Y_{\mathrm{c}}$ and $I$ and the extrapolation to $Y_{\mathrm{c}} \cong 0$ as $I \rightarrow 0$ seen in Figure 4 support the assertion made in the Introduction regarding nonalteration of micelle composition by the polyelectrolyte. This assumption of uniform mixed micelle intramicellar composition could be questioned if the micelle were polarized in the presence of the polyelectrolyte, leading to micelles with an effective local composition $y_{\text {local }}>Y$ that bind more strongly than would be expected based on the stoichiometric $Y$. A consequence of $y_{\text {local }}$ $>Y$ would be diminution in the observed $Y_{\mathrm{c}}$ values, particularly at low $Y$. As a result, extrapolation of $Y_{\mathrm{c}}$ values to zero as $I \rightarrow$ 0 , as seen in Figure 4, would be unlikely. Polarization of the micelle would require that the interaction with the polyelectrolyte overcome the usual headgroup repulsions and entropy that favor intramicellar homogeneity; however, since $Y=Y_{\mathrm{c}}$ is the

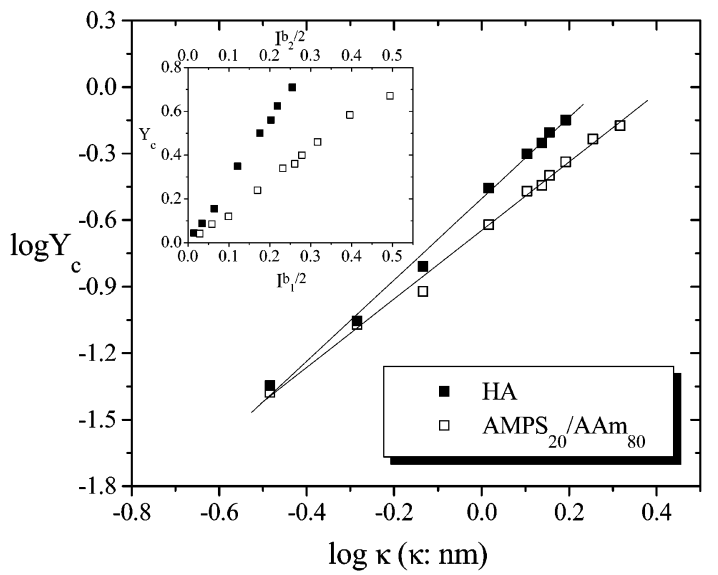

Figure 5. $\log Y_{\mathrm{c}}-\log \kappa$ plot. Inset: $Y_{\mathrm{c}}$ versus $I^{b / 2}$ (as suggested by eq 2 ), with $b_{1}=1.83$ for HA (ם) and $b_{2}=1.53$ for $\operatorname{AMPS}_{20} / \mathrm{AAm}_{80}(\square)$, as empirical fitting parameter.

TABLE 2: Polyelectrolyte Chain Parameters and Empirical Constant $b$ in Eq 2 for the Case of Spherical Micelles

\begin{tabular}{|c|c|c|c|}
\hline & $l_{\mathrm{p}}^{\circ}, \mathrm{nm}$ & $\xi$ & b \\
\hline DNA & $50^{a}$ & 3 & $1.6^{b}$ \\
\hline NaPSS & $3^{c}$ & 3 & $1.4^{d}$ \\
\hline $\mathrm{AMPS}_{20} / \mathrm{AAm}_{80}$ & $2.9^{e}$ & 0.6 & $1.5^{f}$ \\
\hline HA & $4^{g}$ & 0.6 & $1.8^{f}$ \\
\hline
\end{tabular}

${ }^{a}$ From ref $36 a .{ }^{b}$ From ref $36 \mathrm{~b} .{ }^{c}$ From ref $37 \mathrm{a} .{ }^{d}$ From ref $37 \mathrm{~b}$. ${ }^{e}$ From ref 25. ${ }^{f}$ Current study. ${ }^{g}$ From ref 28.

condition of incipient binding, the polyelectrolyte-micelle interaction then is barely larger than $k T$, and the binding energy is insufficient for micelle reorganization.

Theoretical and experimental studies indicate that the critical colloid surface charge density for the binding of polyelectrolytes to oppositely charged colloids may be expressed as

$$
\sigma_{\text {crit }} \xi \sim \kappa^{b} \sim I^{b / 2}
$$

where $\kappa$ is the Debye-Hückel parameter $\left(\kappa^{-1}\right.$, the Debye length in $\mathrm{nm}$, is very nearly $0.3 / I^{1 / 2}$ ), and the scaling term $b$ is reported as 3,1 , and $11 / 5$ in refs 12,13 , and $14 \mathrm{a}$, respectively. For spherical surfaces, Muthukumar reported a slightly different value of $b$ than in the case of planar surfaces. ${ }^{14 \mathrm{~b}}$ Since we can approximate $\mathrm{d} \sigma / \mathrm{d} \kappa$ by $\mathrm{d} Y / \mathrm{d} \kappa$, we plot in Figure $5 \log Y_{\mathrm{c}}$ vs $\log$ $\kappa$. As noted above, data for HA at $I>300 \mathrm{mM}$ are not used, to ensure that micelles have similar dimensions for $\mathrm{HA}$ and copolymer in the measured $Y$ range. Linear fitting leads to slopes (corresponding to $b$ in eq 2) of $1.83 \pm 0.01$ for HA and $1.55 \pm$ 0.01 for $\mathrm{AMPS}_{20} / \mathrm{AAm}_{80}$. As seen in Table 2, these values of $b$ are consistent with those found for other polyelectrolyte-micelle systems. $b$ appears to be sensitive to both the polymer linear charge density and the polymer chain stiffness, expressed by the bare persistence length $\left(l_{\mathrm{p}}{ }^{\circ}\right)$. Comparison between NaPSS and $\mathrm{AMPS}_{20} / \mathrm{AAm}_{80}$ (both having nearly the same bare persistence lengths but different charge densities) and between HA and $\mathrm{AMPS}_{20} / \mathrm{AAm}_{80}$ (both having similar charge densities but different persistence lengths) is instructive, and indicates the dominant effect of persistence length on the value of $b$.

Muthukumar ${ }^{14 \mathrm{~b}, \mathrm{c}}$ considered the effect of $l_{\mathrm{p}}$ on the binding of a polyelectrolyte of repeat unit charge $q$ onto a uniformly charged spherical surface of radius $R$ and obtained the result that - at constant $I, q$, and $R-\sigma_{\text {crit }} \sim l_{\mathrm{K}} \alpha^{2}$, where $l_{\mathrm{K}}$ is the Kuhn length and $\alpha^{2}$ is the expansion factor for the mean-square end-to-end distance. Consequently, we can write for HA and 
$\mathrm{AMPS}_{20} / \mathrm{AAm}_{80}$

$$
\frac{\sigma_{\mathrm{c}}^{\mathrm{HA}}}{\sigma_{\mathrm{c}}^{\mathrm{AA}}}=\frac{\left(l_{\mathrm{K}} \alpha^{2}\right)^{\mathrm{HA}}}{\left(l_{\mathrm{K}} \alpha^{2}\right)^{\mathrm{AA}}} \cong 1.38 \frac{\left(\alpha^{2}\right)^{\mathrm{HA}}}{\left(\alpha^{2}\right)^{\mathrm{AA}}}
$$

where the superscript AA denotes the copolymer of $\mathrm{AMPS}_{20} /$ AAm $_{80}$. References 25 and 28 give values for $\alpha$ as a function of ionic strength for $\mathrm{AMPS}_{17} / \mathrm{AAm}_{83}$ and $\mathrm{HA}$, respectively. ${ }^{38}$ These values lead via eq 3 to predicted ratios for $Y_{\mathrm{c}}^{\mathrm{HA}} / Y_{\mathrm{c}} \mathrm{AA}$ of $1.5 \pm 0.2$, in good agreement with the experimental values from Table 1 in the range of $0.05 \mathrm{M}<I<0.3 \mathrm{M}$, but with no clear ionic strength dependence.

The bare persistence length $l_{\mathrm{p}}^{\circ}$ clearly does not alone determine the difference between micelle binding of the two polymers, since that difference would be maintained at low $I$, where in fact the difference in $Y_{\mathrm{c}}$ disappears. The data in refs 25 and 28 furthermore show that neither the total persistence length $\left(l_{\mathrm{p}}^{\mathrm{T}}\right)$ nor $l_{\mathrm{K}} \alpha^{2}$ is seen to converge on 1 at low $I$, even though the experimental values of $Y_{\mathrm{c}}{ }^{\mathrm{HA}} / Y_{\mathrm{c}}{ }^{\mathrm{AA}}$ do. However, all available theoretical ${ }^{12-15}$ and empirical ${ }^{2,9,37 b, 39,40}$ expressions for $\sigma_{\text {crit }}$ (hence $Y_{\mathrm{c}}$ ) suggest that the convergence of $Y_{\mathrm{c}}$ at constant $\xi$, $\kappa$, and $R$ can occur only if the polymer chains have equal stiffness. Therefore, we consider some other stiffness parameter that might show the expected convergence as $I \rightarrow 0$. A rationale for seeking a stiffness parameter that weighs the contribution of electrostatic expansion term to the total persistence length less than the usual Odijk-Skolnick-Fixman formulation ${ }^{41,42}$ is provided by the expectation that intrapolymer electrostatic stiffening must be reduced in the potential domain of the oppositely charged colloid relative to what occurs when the polyion experiences only its own electrostatics. A similar phenomenon is considered to be responsible for the observation that polyelectrolyte-micelle binding shows no special feature or discontinuity at $\xi=1$, the Manning counterion condition. ${ }^{24 a}$ Such a stiffness parameter is provided by an empirical "effective persistence length":

$$
l_{\mathrm{p}}^{\mathrm{eff}} \equiv l_{\mathrm{p}}^{\circ}+\frac{1}{4} l_{\mathrm{p}}^{\mathrm{el}}
$$

where $l_{\mathrm{p}}$ el is the electrostatic persistence length. The fraction $1 / 4$ in eq 4 is an empirical parameter which is found by trialand-error fitting of the $l_{\mathrm{p}}$ eff vs $I$ graphs in order to replicate the experimental results of both convergence at low salt concentration and divergence at high salt. In a recent theoretical treatment of the effect of persistence length on polyelectrolyte-colloid interaction, ${ }^{24 \mathrm{~b}}$ in which the reduction of the bending energy of the polyelectrolyte complexed with a colloid of opposite charge is treated as a consequence of the neutralization of fraction $\alpha$ of polymer charges, the coefficient $1 / 4$ in eq 4 would correspond to $\alpha=0.5$. The dependence on $I$ of $l_{\mathrm{p}}$ eff is plotted semilogarithmically and compared to the ionic strength dependence of $Y_{\mathrm{c}}$ in Figure 6.

As seen in Figure $6, l_{\mathrm{p}}^{\text {eff }}$ converges for HA and the copolymer at low $I$ in the same way as $Y_{\mathrm{c}}$ does. This supports the suggestion that some chain stiffness parameter intermediate between $l_{\mathrm{p}}{ }^{\mathrm{T}}$ and $l_{\mathrm{p}}{ }^{\circ}$ controls the binding. The convergence of values at low ionic strength may be understood by the predominant effect of $l_{\mathrm{p}}^{\mathrm{el}}$, making both chains stiffer. As the salt concentration is increased, the $Y_{\mathrm{c}}$ values start to diverge due to the effect of the higher value of $l_{\mathrm{p}}{ }^{\circ}$ for HA than for the copolymer (4.0 vs 3.0 $\mathrm{nm})$. While an increase in ionic strength enhances the overall flexibility of the polymer as expressed by a decrease in persistence length, binding is always weakened (an increase in

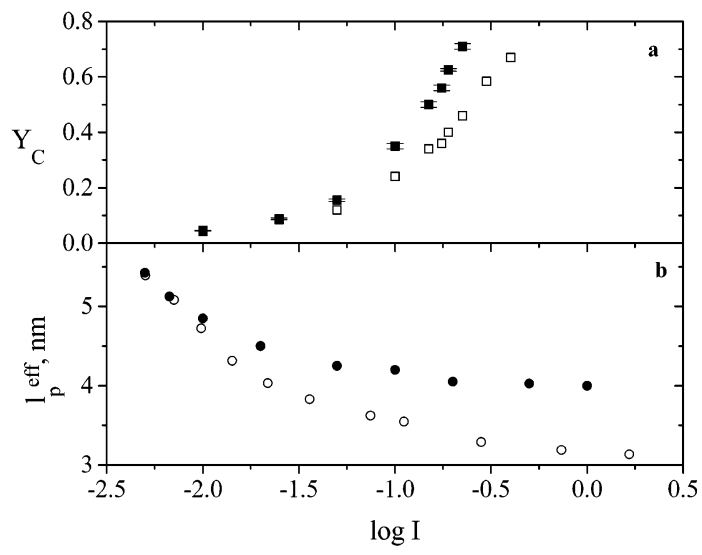

Figure 6. (a) $Y_{\mathrm{c}} \mathrm{vs} \log I$ for HA and $\mathrm{AMPS}_{20} / \mathrm{AAm}_{80}$. (b) $l_{\mathrm{p}}^{\text {eff }}$ vs $\log$ $I$, based on $l_{\mathrm{p}}^{\mathrm{T}}$ and $l_{\mathrm{p}}{ }^{\circ}$ in $l_{\mathrm{p}}{ }_{\mathrm{p}}^{\text {eff }}$ values from refs 28 and 25 . Filled symbols for HA and open symbols for AMPS ${ }_{20} / \mathrm{AAm}_{80}$.

$\left.Y_{\mathrm{c}}\right)$ with added salt. This is because the primary effect of added salt is to diminish the electrostatic attraction between the cationic micelle and the anionic polymer.

Polyelectrolyte-Protein Interactions. Polyelectrolytes bind to proteins through electrostatic interactions, but the asymmetry and heterogeneity of protein charge distributions means that the binding site on the protein can be localized. Because glycosaminoglycans are among the few examples of flexible chain polyelectrolytes in mammals, and because their binding to proteins is recognized as having considerable biochemical significance, ${ }^{43}$ we consider the binding of serum albumin (SA) to hyaluronic acid (HA) and to another glycosaminoglycan, heparin (Hp), which has a higher charge density and lower persistence length. While neither of these systems represents a biochemical cognate pair, there has been some consideration of the possible consequences of SA-HA interactions in synovial fluid. ${ }^{32}$ To further evaluate the effects of chain stiffness and charge density, the results for $\mathrm{HA}-\mathrm{SA}$ and $\mathrm{Hp}-\mathrm{SA}$ were compared with the binding of SA to two synthetic polyelectrolytes, copolymers $\mathrm{AMPS}_{25} / \mathrm{AAm}_{75}$ and $\mathrm{AMPS}_{80} / \mathrm{AAm}_{20}$, the first of which has a mean charge density equal to that of HA, while the second has an average charge density similar to that of Hp. Both bovine serum albumin (for $\mathrm{AMPS}_{25} / \mathrm{AAm}_{75}$, $\mathrm{AMPS}_{80} / \mathrm{AAm}_{20}, \mathrm{Hp}$, and HA interaction) and human serum albumin (for HA interaction) were used for this study, and the results are represented without differentiation since both proteins revealed the same results regarding critical binding conditions. ${ }^{46}$

Since protein charges are $\mathrm{pH}$ dependent, titrations were carried out by varying $\mathrm{pH}$ and observing the turbidity change in order to identify a critical $\mathrm{pH}$ at which complex formation begins. The ionic strength dependence of $\mathrm{pH}_{\mathrm{c}}$ is a "phase boundary" that can be more meaningfully represented by the ionic strength dependence of the net protein charge at conditions of incipient binding $\left(Z_{\mathrm{c}}\right)$. The net protein charge is calculated by summing up the amino acid charges determined using the spherical-smeared-charged model put forward by Tanford, ${ }^{47 a}$ utilizing the protein titration curve for $\mathrm{BSA}^{47 \mathrm{~b}}$ as explained previously. ${ }^{44}$ The plot of $Z_{\mathrm{c}}$ vs $I$ is shown in Figure 7. In contrast to $Y_{\mathrm{c}}$, the net protein charge does not clearly represent the subtle combination of attraction arising from a local positive charge and repulsion arising from neighboring negative sites. ${ }^{44}$ Put differently, $Z=0$, i.e., the isoelectric point, has no significance with regard to polyanion binding which can occur on both sides of the isoelectric point. It should also be noted that values of $\mathrm{pH}_{\mathrm{c}}$ below 5 (i.e., $Z_{\mathrm{c}}<0$ ) correspond to protonation of the carboxylate groups of HA, complicating the interpretation of the phase boundary. 


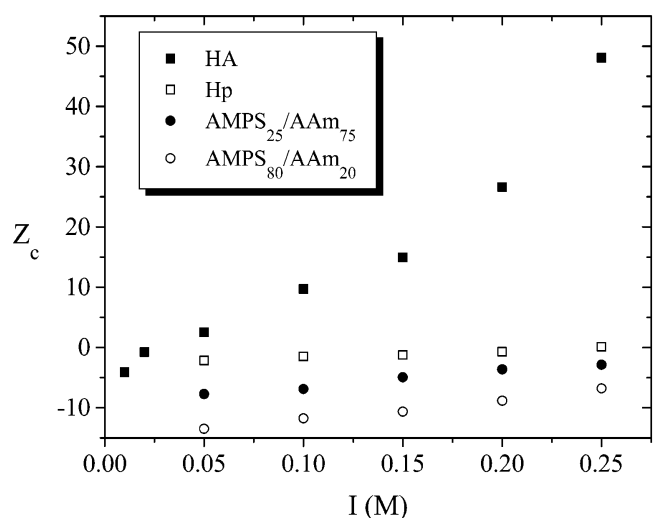

Figure 7. Ionic strength dependence of protein net charge at critical

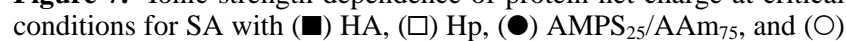
$\mathrm{AMPS}_{80} / \mathrm{AAm}_{20}$.

Although $\mathrm{HA}$ and $\mathrm{AMPS}_{25} / \mathrm{AAm}_{75}$ have similar linear charge densities, the binding of SA to $\mathrm{AMPS}_{25} / \mathrm{AAm}_{75}$ occurs readily when $Z_{\mathrm{c}}$ is well below zero, while the onset of HA binding occurs at more positive values of $Z_{\mathrm{c}}$, with $Z_{\mathrm{c}}>0$ at higher ionic strengths. The greater flexibility of $\mathrm{AMPS}_{25} / \mathrm{AAm}_{75}$ can be expected to facilitate polymer configurations that will reduce the repulsive effect arising from negatively charged protein domains and favor the alignments around the positively charged protein domains. ${ }^{44}$ The stiffness of HA corresponds to a restriction of chain movements, so that the bound configuration may not readily avoid negative domains, and therefore binding cannot take place when the net charge is strongly negative. As seen in the micelle-binding case discussed above, the difference between flexible and semiflexible polyions increases at high salt, while the phase boundaries converge at low salt. As noted before, we ascribe this to the electrostatic contribution to $l_{\mathrm{p}}$ which confers on both chains similar stiffness at low salt, while the behavior at high salt reflects the difference in bare persistence lengths.

The weak binding of HA can be understood on the basis of its low charge density and high persistence length. The differences in binding affinities among the other three polymers are more complex. We consider first the low protein affinity of $\mathrm{Hp}$, whose charge density is similar to that of $\mathrm{AMPS}_{80} / \mathrm{AAm}_{20}$ and much higher than that of $\mathrm{AMPS}_{25} / \mathrm{AAm}_{75}$. A possible explanation for this may reside in the variety of sequences of charged and uncharged residues available to the random copolymers. We can imagine some particular sequences complementary to the charge pattern on the protein at the binding site, particularly a run of anionic residues bracketed by nonionic ones, complementary to a positive patch located among negatively charged domains. Since $\mathrm{pH}_{\mathrm{c}}$, and hence $Z_{\mathrm{c}}$, corresponds to the onset of binding, such high-protein-affinity sites can have a disproportionate influence on the point of incipient complex formation. In contrast, all $\mathrm{Hp}$ disaccharides bear some charge $\mathrm{e}^{45}$ with an average of 3.4 charges per disaccharide; therefore, a segment with consecutive uncharged units is not probable for Hp. This hypothesis could be tested by separating high- and low-protein-affinity components of the copolymer distribution and comparing compositions.

\section{Conclusion}

The effect of chain stiffness on the interaction of polyelectrolytes $\mathrm{HA}$ and $\mathrm{AMPS}_{20} / \mathrm{AAm}_{80}$ with oppositely charged colloidal particles has been studied by measuring the surface charge density of a cationic-nonionic mixed micelle at which one observes a transition from noninteracting to complex states.
The stiffer chain was observed to bind more weakly, the effect being more evident at higher ionic strengths at which condition the difference in chain stiffness is dominated by the difference in bare persistence lengths. The interaction of $\mathrm{HA}$ and $\mathrm{AMPS}_{25} /$ $\mathrm{AAm}_{75}$ with serum albumin shows a more striking binding affinity for the more flexible chain due to its ability to adopt configurations that bind to a local positive "patch", while minimizing the repulsive effect arising from the negatively charged domains of the protein. The nature of the polyelectrolyte charge distribution may also have a significant effect on interactions with proteins as inferred from differences among $\mathrm{Hp}, \mathrm{AMPS}_{25} / \mathrm{AAm}_{75}$, and AMPS $80 / \mathrm{AAm}_{20}$. Thus, it is proposed that the low binding of $\mathrm{Hp}$ is due to the absence of nonionic residues that could reduce the repulsive effect when a bound sequence is forced to partially interact with negatively charged domains of the protein.

Acknowledgment. Support from the National Science Foundation under Grants CHE9987891 and DMR0076068 is acknowledged.

\section{References and Notes}

(1) Dubin, P. L.; Curran, M. E.; Hua, J. Langmuir 1990, 6, 707.

(2) McQuigg, D. W.; Kaplan, J. I.; Dubin, P. L. J. Phys. Chem. 1992, 96, 1973

(3) Dubin, P. L.; Rigsbee, D. R.; McQuigg, D. W. J. Colloid Interface Sci. 1985, 105, 509.

(4) Dubin, P. L.; Rigsbee, D. R.; Gan, L. M.; Fallon, M. A. Macromolecules 1988, 21, 2555.

(5) Li, Y.; Xia, J.; Dubin, P. L. Macromolecules 1994, 27, 7049.

(6) Dubin, P. L.; Thé, S. S.; Gan, L. M.; Chew, C. H. Macromolecules 1990, 23, 2500.

(7) Dubin, P. L.; Thé, S. S.; McQuigg, D. W.; Gan, L. M.; Chew, C. H. Langmuir 1989, 5, 89 .

(8) (a) Lindman, B.; Thalberg, K. Polymer-Surfactant Interactions: Recent Developments. In Interactions of Surfactants with Polymers and Proteins; Goddard, E. D., Ananthapadmanabhan, K. P., Eds; CRC Press: Boca Raton, FL, 1993. (b) Thalberg, K.; Lindman, B. J. Phys. Chem. 1989, 93, 1478. (c) Kiefer, J.; Somasundaran, P.; Ananthapadmanabhan, K. P. Langmuir 1993, 9, 1187. (d) Hansson, P.; Almgren, M. J. Phys. Chem. 1996, 100, 9038.

(9) Polyelectrolytes for Water and Wastewater Treatment; Schwoyer, W. L. K., Ed.; CRC Press: Boca Raton, FL, 1981.

(10) Shaner, S. L.; Melancon, P.; Lee, K. S.; Burgess, M. T.; Record, M. T., Jr. Cold Spring Harbor Symp. Quant. Biol. 1983, 47, 463.

(11) von Hippel, P. H.; Bear, D. G.; Morgan, W. D.; McSwiggen, J. A. Annu. Rev. Biochem. 1984, 53, 389.

(12) Weigel, F. W. J. Phys. A: Math. Gen. 1977, 10, 299.

(13) Evers, O. A.; Fleer, G. J.; Scheutjens, J. M. H. M.; Lyklema, J. J. Colloid Interface Sci. 1986, 111, 446.

(14) (a) Muthukumar, M. J. Chem. Phys. 1987, 86, 7230. (b) von Goeler, F.; Muthukumar, M. J. Chem. Phys. 1994, 100, 7796. (c) Kong, C. Y.; Muthukumar, M. J. Chem. Phys. 1998, 109, 1522.

(15) Odijk, T. Macromolecules 1980, 13, 1542.

(16) Feng, X. H.; Dubin, P. L.; Zhang, H.; Kirton, G. F.; Bahadur, P.; Parotte, J. Macromolecules 2001, 34, 6373.

(17) (a) Park, J.; Muhoberac, B. B.; Dubin, P. L.; Xia, J. Macromolecules 1992, 25, 290. (b) Xia, J.; Dubin, P. L.; Kim, Y.; Muhoberac, B. B.; Klimkowski, V. J. J. Phys. Chem. 1993, 97, 4528.

(18) (a) Li, Y.; Mattison, K.; Dubin, P. L.; Havel, H.; Edwards, S Biopolymers 1996, 38, 527. (b) Mattison, K. M.; Brittain, I. J.; Dubin, P. L. Biotechnol. Prog. 1995, 11, 632. (c) Mattison, K. W.; Dubin, P. L.; Brittain, I. J. J. Phys. Chem. B 1998, 102, 3830.

(19) Wallin, T.; Linse, P. Langmuir 1996, 12, 305.

(20) Wallin, T.; Linse, P. J. Phys. Chem. 1996, 100, 17873.

(21) Skepo, M.; Linse, P. Phys. Rev. E 2002, 66, 51807.

(22) Stoll, S.; Chodanowski, P. Macromolecules 2002, 35, 9556.

(23) Norisuye, T. Hydrocolloids 2000, 2, 311.

(24) (a) Manning, G. S. J. Chem. Phys. 1969, 51, 924. (b) Manning, G. S. J. Phys. Chem., submitted for publication.

(25) Reed, W. F.; Ghosh, S.; Medjahdi, G.; François, J. Macromolecules 1991, 24, 6189.

(26) Cleland, R. L. Biopolymers 1984, 23, 647. 
(27) Hayashi, K.; Tsutsumi, K.; Nakajima, F.; Norisuye, T.; Teramoto, A. Macromolecules 1995, 28, 3824.

(28) Hayashi, K.; Tsutsumi, K.; Nakajima, F.; Norisuye, T.; Teramoto, A. Polym. J. 1996, 28, 922.

(29) Fouissac, E.; Milas, M.; Rinaudo, M.; Borsali, R. Macromolecules 1992, 26, 5613 .

(30) Ghosh, S.; Li, X.; Reed, C.; Reed, W. Biopolymers 1990, 30, 1101.

(31) Carlsonn, F.; Malmsten, M.; Linse, P. J. Phys. Chem. B 2001, 105, 12189 .

(32) (a) Moskowitz, R. W.; Howell, D. S.; Goldberg, V. M.; Mannkin,

H. J. Osteoarthritis: Diagnosis and Medical/Surgical Management, 2nd ed.; W. B. Saunders: Philadelphia, PA, 1992; p 200 ff. (b) Olsten, A. G.; Stonier, J. E. Biochem. J. 1952, 52, 149. (c) Palfrey, A. J.; White, J. B. Biorheology 1968, 5, 189.

(33) Gao, J. Y.; Dubin, P. L.; Sato, T.; Morishima, Y. J. Chromatogr., A 1997, 766, 233.

(34) Davis, R. M.; Zhang, H.; Dubin, P. L.; McQuigg, D. W. Polym. Prepr. (Am. Chem. Soc., Div. Polym. Chem.) 1991, 32, 292.

(35) Li, Y. J.; Xia, J. L.; Dubin, P. L. Macromolecules 1994, 27, 7049.

(36) (a) Cantor, C. R.; Schimmel, P. R. Biophysical Chemistry Part III; W. H. Freeman and Co.: New York, 1980; p 1033. (b) Wang, Y.; Dubin, P. L.; Zhang, H. Langmuir 2001, 17, 1670.
(37) (a) Hattori, T.; Hallberg, R.; Dubin, P. L. Langmuir 2000, 16, 9738. (b) Zhang, H.; Ohbu, K.; Dubin, P. L. Langmuir 2000, 16, 9082. 28. (38) $\alpha$ is obtained as $\left([\eta] /[\eta]_{\theta}\right)^{1 / 3}$ from the viscosity data reported in ref

(39) Zhang, H.; Dubin, P. L.; Ray, J.; Manning, G. S.; Moorefield, C. N.; Newkome, G. R. J. Phys. Chem. B 1999, 103, 2347.

(40) Miura, N.; Dubin, P. L.; Moorefield, C. N.; Newkome, G. R. Langmuir 1999, 15, 4245.

(41) Odijk, T. J. Polym. Sci., Polym. Phys. Ed. 1977, 15, 477.

(42) Skolnick, J.; Fixman, M. Macromolecules 1977, 10, 944.

(43) Jackson, R. L.; Busch, S. J.; Cardin, A. D. Phys. Rev. 1991, 71, 481 .

(44) Seyrek, E.; Tribet, C.; Dubin, P. L.; Gamble, E. A. Biomacromolecules 2003, 4, 273.

(45) Casu, B. Adv. Carbohydr. Chem. Biochem. 1985, 43, 51.

(46) Staggemeier, B. A. Studies of Protein-Polyelectrolyte Binding: A Comparison of Biological and Synthetic Polyelectrolytes. M.S. Thesis, IUPUI, 1999.

(47) (a) Hattori, T.; Kimura, K.; Seyrek, E.; Dubin, P. L. Anal. Biochem 2001, 158, 158. (b) Tanford, C.; Kirkwood, J. G. J. Am. Chem. Soc. 1957, 79, 5333. (c) Tanford, C.; Swanson, S. A.; Shore, W. S. J. Am. Chem. Soc. 1955, 77, 6414 